\title{
ADAMTS-1 is involved in normal follicular development, ovulatory process and organization of the medullary vascular network in the ovary
}

\author{
M Shozu ${ }^{1}$, N Minami ${ }^{1}$, H Yokoyama ${ }^{2}$, M Inoue ${ }^{1}$, H Kurihara $^{3}$, K Matsushima ${ }^{4}$ and K Kuno ${ }^{5}$ \\ Department of ${ }^{1}$ Obstetrics and Gynecology, ${ }^{2}$ Division of Blood Purification, School of Medicine, Kanazawa University, 13-1 Takara-machi, Kanazawa, Ishikawa, 920-0934 Japan, \\ Department of ${ }^{3}$ Physiological Chemistry and Metabolism, ${ }^{4}$ Molecular Preventive Medicine, Graduate School of Medicine, University of Tokyo, 7-3-1 Hongo, Bunkyo-ku, \\ Tokyo, 113-0033 Japan and ${ }^{5}$ Center for the Development of Molecular Target Drugs, Cancer Research Institute, Kanazawa University, 13-1 Takara-machi, Kanazawa, \\ Ishikawa, 920-0934 Japan
}

(Requests for offprints should be addressed to K Kuno; Email: kkuno@kenroku.kanazawa-u.ac.jp)

\begin{abstract}
To clarify the role of disintegrin-like and metalloproteinase with thrombospondin type I motifs-1 (ADAMTS-1) in ovarian function, we examined abnormalities in ovulatory processes, folliculogenesis and the vascular system of ADAMTS-1 null ovaries. First, when immature female mice were treated with pregnant mare serum gonadotropin (PMSG)/human chorionic gonadotropin ( $\mathrm{hCG}$ ), the number of ovulated oocytes was markedly decreased in ADAMTS-1 null mice in comparision to ADAMTS-1 (+/-) controls. The proportion of anovulated follicles to total mature follicles was significantly higher in ADAMTS-1 null females when compared with controls. The numbers of growing follicles at each stage were counted. The number of follicles at type $5 \mathrm{~b}$ (late preantral) and later stages was markedly reduced in ADAMTS-1 null mice, irrespective of gonadotropin treatment (no gonadotropins, PMSG alone or PMSG/hCG). These data demonstrate that impairment of ovarian function to ovulate oocytes in ADAMTS-1 null mice occurs at two different levels: in the development of growing follicles and ovulatory processes. Furthermore, ADAMTS-1 null ovaries included a number of unusual atretic follicles that showed no sign of oocyte degeneration but lost the surrounding granulosa cell layers and were considered to be derived from type 4 or 5 a follicles. These results suggest that ADAMTS- 1 is important for follicular development beyond the type 4 and/or $5 \mathrm{a}$ and for maintaining normal granulosa cell layers in follicles. Finally, the number of large blood vessels in the medullar zone was significantly decreased in ADAMTS-1 null mice ovaries, suggesting that ADAMTS-1 is also involved in the organization of the medullary vascular network.
\end{abstract}

Journal of Molecular Endocrinology (2005) 35, 343-355

\section{Introduction}

ADAMTS-1 is the first member of the disintegrin-like and metalloproteinase with thrombospondin type I motifs (ADAMTS) family, and is characterized by having an ADAM metalloproteinase domain and thrombospondin type I motifs (Kuno et al. 1997a, 1997b). ADAMTS-1 was originally identified as a gene strongly expressed in cachexigenic tumors (Kuno et al. 1997b). ADAMTS-1 consists of proprotein-, metalloproteinaseand disintegrin-like domains, thrombospondin type I motifs and intervening spacer region among them.

As a metalloproteinase associated with the extracellular matrix (ECM) (Kuno \& Matsushima 1998, Kuno et al. 1999), ADAMTS-1 cleaves aggrecan and versican (Kuno et al. 2000, Sandy et al. 2001, RodriguezManzaneque et al. 2002). ADAMTS-1 also possesses anti-angiogenic activity (Vazquez et al. 1999, Luque et al. 2003) and ADAMTS-1 or its C-terminal half region suppresses tumor growth and experimental metastatic potential (Iruela-Arispe et al. 2003, Kuno et al. 2004).
The physiological functions of ADAMTS-1 have been investigated using gene knockout mice produced in our laboratories (Shindo et al. 2000). ADAMTS-1 null mice display renal anomalies involving enlarged calices and atrophic renal papillae, which resemble ureteropelvic junction (UPJ) obstruction in humans (Shindo et al. 2000). This finding indicates that ADAMTS-1 plays an important role in tissue architecture and function in ureteropelvic junction tissue.

We demonstrated that the second major phenotype of ADAMTS-1 null mice is female infertility featuring a markedly low delivery rate and number of pups, indicating that ADAMTS- 1 is important in the function of female reproductive organs (Shindo et al. 2000). Decreased number of implantation sites was also reported in ADAMTS-1 null mice (Shindo et al. 2000, Mittaz et al. 2004). ADAMTS-1 mRNA expression is induced in the granulosa cells of the preovulatory follicles after administration of luteinizing hormone (LH) (Espey et al. 2000, Robker et al. 2000, Boerboom et al. 2003), and is sustained in a progesterone-dependent manner (Robker et al. 2000). The ADAMTS-1 protein 
increases after an LH surge and is localized in the cumulus-oocyte complex (COG) of the preovulatory follicles (Russell et al. 2003). Recently, Mittaz et al. (2004) reported that ADAMTS-1 null mice trap mature oocytes in ovarian follicles, suggesting that ADAMTS-1 is required for normal ovulation.

In the present study, we conducted a detailed examination of the ovarian morphology of ADAMTS-1 null female mice and showed that impairment of ovulation function in ADAMTS-1 null mice can be attributed not only to the to the ovulatory process of mature follicles but also to changes in the growth process of immature follicles.

\section{Materials and methods}

\section{Materials}

Pregnant mare serum gonadotropin (PMSG) and human chorionic gonadotropin (hGG) were purchased from Teikokuzouki Pharmaceutical Co., (Tokyo, Japan)

\section{Animals and hormone treatment}

ADAMTS-1 null mice were generated as described previously (Shindo et al. 2000). Littermates obtained by mating heterozygous females (+/-) with homozygous males (-/-) with the genetic background of the 129/Sv x C57BL/6 hybrid were used for phenotypic analysis. Animal experiments complied with the standards stipulated by the Takara-machi Campus of Kanazawa University and adhered to the principles of the UFAW Handbook on the Care and Management of Laboratory Animals, 7th edition.

\section{Measurement of ovulated oocytes}

Female mice at 24-26 days old were injected i.p. with 7.5 IU of PMSG followed by 7.5 IU of hCG i.p. $48 \mathrm{~h}$ later. Oviducts and ovaries were excised from mice $15 \mathrm{~h}$ after hCG administration and were placed into dishes containing PBS. COC was recovered by dissection of both the ampulla and the oviduct, and under a dissecting microscope, the number of ovulated oocytes was determined after treatment with 500 units $/ \mathrm{ml}$ hyaluronidase (Sigma).

\section{Histological studies and statistical analyses}

Excised ovarian tissues were fixed in 10\% neutral buffered formalin (Wako Pure Chemical, Osaka, Japan) as described previously (Lydon et al. 1995). After the requisite fixation time, ovaries were trimmed, dehydrated with ethanol, cleared in xylene, and infiltrated with paraffin wax.
For analysis of the number of ovarian follicles, each paraffin-embedded ovary was placed with the ovarian hilum at the side and serially sectioned parallel to its longitudinal plane at $2.5 \mu \mathrm{m}$ using a microtome. Every sixth section was mounted and stained with hematoxylin and eosin. Serial sections of ovaries were photographed and printed at x100 magnification, which enabled clear discrimination of each growing follicle. Each follicle was then numbered on the serial photographs and counted only once when the oocyte was seen. Classification and nomination of ovarian follicles were based on Pederson \& Peters (1968). Follicles at the preantral stage were deemed atretic if the oocyte was degenerating (convoluted and condensed or fragmented) (Morita et al. 1999).

Area of medullar zone of each ovary was determined on digital microscopic images of the serial sections using MacSCOPE software (Mitani Co., Fukui, Japan). The medullar zone was defined expediently as the internal area of the ovarian cortex which consists of follicles and surrounding ovarian stroma. The section with the largest medullary area among the serial sections (defined as the central plane) was used as the representative section for comparison. The blood vessel area in the medullary zone was also measured on the central plane using MacSCOPE.

The Mann-Whitney test was used for statistical evaluation and $\mathrm{P}<0.05$ was considered to be statistically significant. Fisher's direct probability test was used for statistical evaluations of frequency of unruptured follicles showing COC expansion and frequency of large follicles having two vascular plexus layers.

\section{Immunohistochemical analyses}

Cryostat sections of ovary tissue were stained with anti-mouse CD31 monoclonal antibody (BectonDickinson, Sunnyvale, CA, USA) with the aid of a HISTOFINE system (Nichirei Co., Tokyo, Japan) as described previously (Kuno et al. 2004).

\section{Results}

\section{Decreased number of ovulated oocytes in ADAMTS-1 null females}

In order to evaluate the total ovarian function of ADAMTS-1 null mice, we first examined the ovulation of oocytes in response to exogenous gonadotropins. Immature mice were used for the study because they ovulate solely in response to exogenous gonadotropins, by which the super-ovulation scheme was applied. We confirmed that ADAMTS- $1+/$ - females were as fertile as $+/+$ mice (data not shown), and thus ADAMTS- 1 $+/$ - female mice were used as controls for assessment of ovarian function. After treatment with PMSG followed 


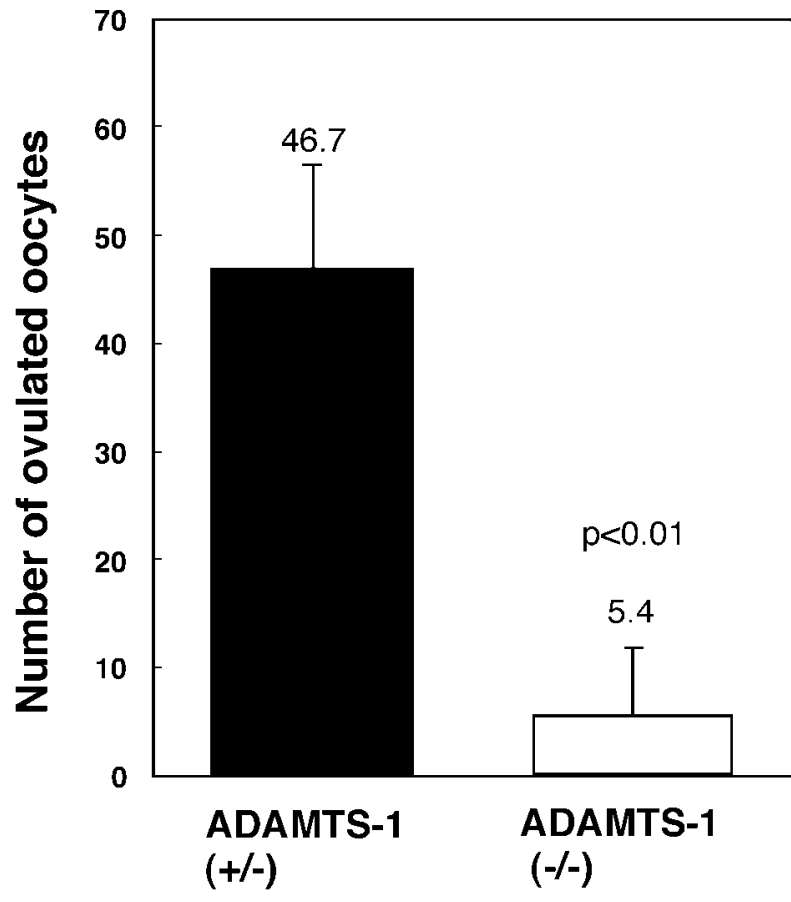

Figure 1 Reduction in the number of ovulated oocytes in ADAMTS-1 knockout mice under super-ovulatory conditions. ADAMTS-1 -/- $(n=8)$ and ADAMTS-1 +/- $(n=9)$ mice were treated with PMSG/hCG, $15 \mathrm{~h}$ after which oocytes were recovered from both oviducts of mice and were counted. The results are expressed as oocytes per mouse. The data are means \pm S.D. and numerical mean value is also indicated.

by hCG, ovulated oocytes in the oviduct were collected and counted. As shown in Fig. 1, ovaries of the ADAMTS-1 null females ovulated one ninth the number of oocytes as control mice. These data suggest that the total ovarian function of ADAMTS-1 null female mice is impaired.

\section{Impaired follicular development in ADAMTS-1 null mice}

To examine whether ADAMTS-1 is involved in the maturation process of follicles, we first compared the number of total mature follicles in immature mice after PMSG/hCG treatment. The numbers of corpus luteum (CL), unruptured follicles (UF, mature follicles that have not undergone ovulation), and luteinized unruputred follicles (LUF, luteinized follicles that trap oocyte inside) per ovary were separately counted for each ovary and the sum of these was defined as the number of total mature follicles (Table 1). As shown in Fig. 2A, the number of total mature follicles in the ovaries of ADAMTS-1 null mice was one-third lower than that of control mice, suggesting that follicular maturation is significantly impaired in ADAMTS-1 knockout mice.

To determine the stages of follicular development affected by disruption of the ADAMTS-1 gene, the number of follicles at follicular stage was compared with controls at three time points, before (no stimulation, Fig. 2B), during (PMSG alone, Fig. 2C) and after PMSG/hCG treatment (Fig. 2D). The results were similar at these time points. The number of type 5b follicles (late preantral follicles with more than four layer of granulosa cells) and higher stage follicles were decreased in ADAMTS-1 null mice, whereas the number of the earlier type 4 follicles (small preantral follicles with only two layers of granulosa cells) were increased in ADAMTS null mice, although the

Table 1 Terms describing the follicular development used in the present study

\section{Description}

\section{Follicle type}

(1) Stages of follicular development based on Pederson \& Peter (1968):

Type 4

Type $5 a$

Type $5 b$

Type 6

(2) Total mature follicles after injection of hCG

UF

$\mathrm{LH}$

$\mathrm{CL}$

Anovulatory follicles

(3) General terms showing the follicular development

Preantral Follicles with more than two

Antral without follicle fluid

UF+LUF

(type 4+type 5a+type 5b)

Follicles with antrums
Early preantral follicles with two layers of granulosa cells

Middle preantral follicles with three layers of granulosa cells

Late preantral follicles with more than four layers of granulosa cells and

Antral follicles with small scattered antrums

Follicles that have finished maturation $(U F+L U F+C L)$

Mature follicles that have not undergone ovulation

Luteinized follicles that have not undergone ovulation and include oocytes

Luteinized follicles that have undergone ovulation and have stigma

UF, unruptured follicles; LUF, luteinized unruptured follicles; CL, corpus luteum. 


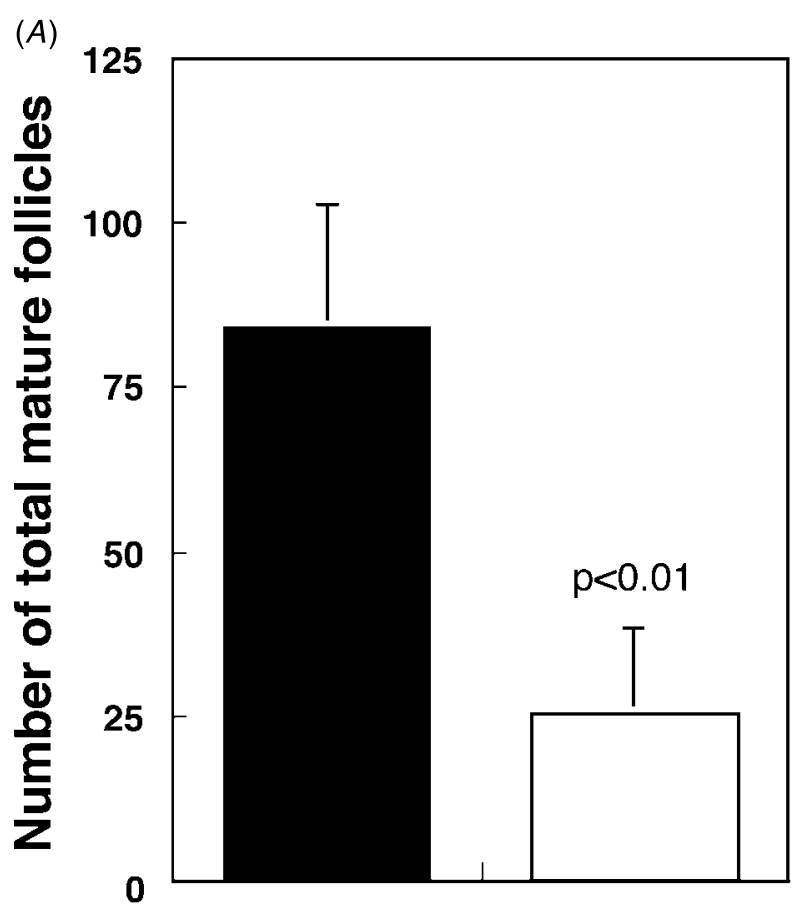

\section{ADAMTS-1 ADAMTS-1 $(+/-)$ $(-/-)$}

(C)

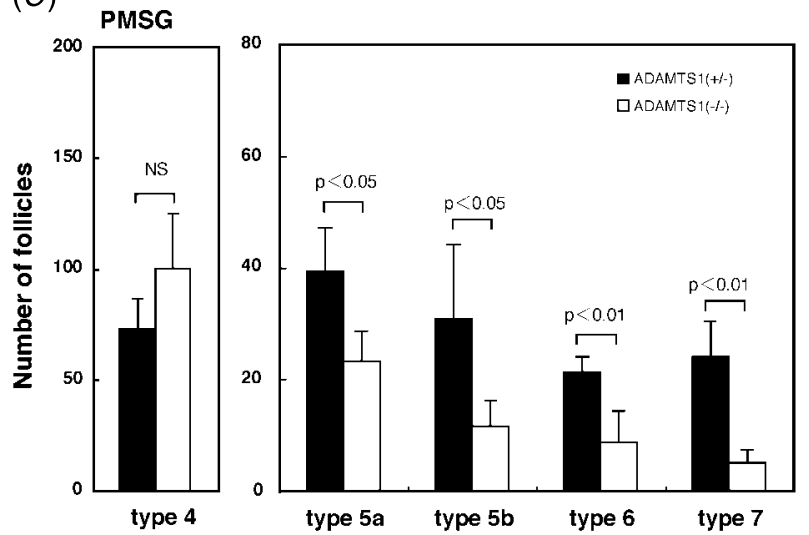

(B)

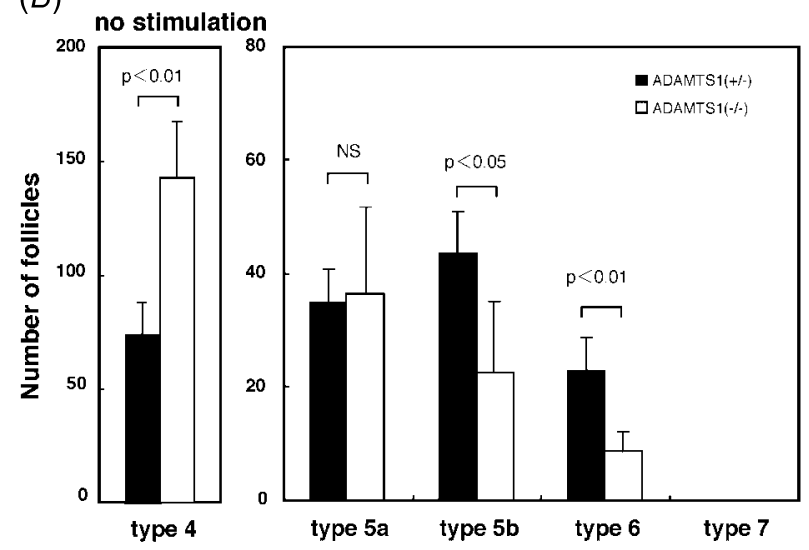

(D)
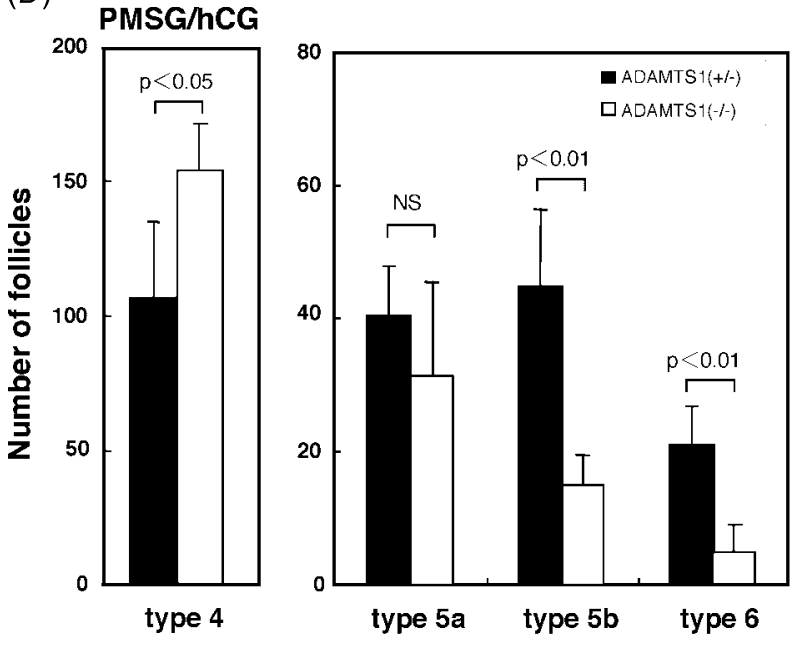

Figure 2 Involvement of ADAMTS-1 in follicular development. (A) Comparison of the number of total mature follicles in super-ovulatory ADAMTS-1 -/- and ADAMTS-1 +/- mice. ADAMTS-1 -/- $(n=7)$ and ADAMTS- $1+/-(n=7)$ mice were treated with PMSG/hCG, followed by excision of ovaries, which were then histologically analyzed. Total mature follicles were calculated as the sum of CL, UF, and LUF. The results are expressed as follicles per ovary. The data are means \pm S.D. (B-D) Numbers of ovarian follicles at different stages in ADAMTS-1 /- and +/- mice treated with PMSG (C), or PMSG/hCG (D), and without gonadotropin (B). ADAMTS-1 -/- and ADAMTS-1 +/- mice were treated with PMSG (48 h) ( $\mathrm{n}=5$ for each group) (C), or PMSG (48 h) /hCG (15 h) ( $n=6$ for each group) (D), and without gonadotropin ( $n=5$ for each group) (B), followed by excision of ovaries, which were then histologically analyzed. Staging of ovarian follicles is based on the classification of Pederson and Peters (1968). The results are expressed as follicles per ovary. The data are means \pm S.D.

difference was not sufficient to reach significance in mice treated with PMSG alone. For type 5a follicles (small preantral follicles with three layers of granulosa cells), the difference was barely significant in mice treated with PMSG alone (Fig. 2C). These results demonstrate that follicular development is significantly impaired at type $5 \mathrm{~b}$ and thereafter in pre-pubertal ADAMTS- 1 null mice, independent of the exogenous gonadotropin 


\section{(A)}

no stimulation

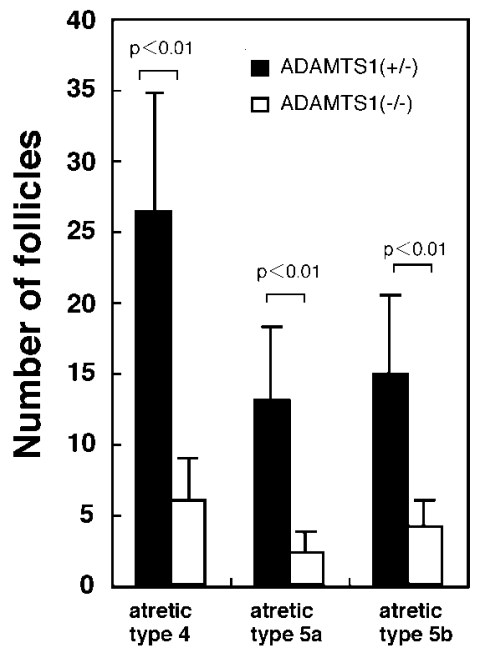

(B)

(C)

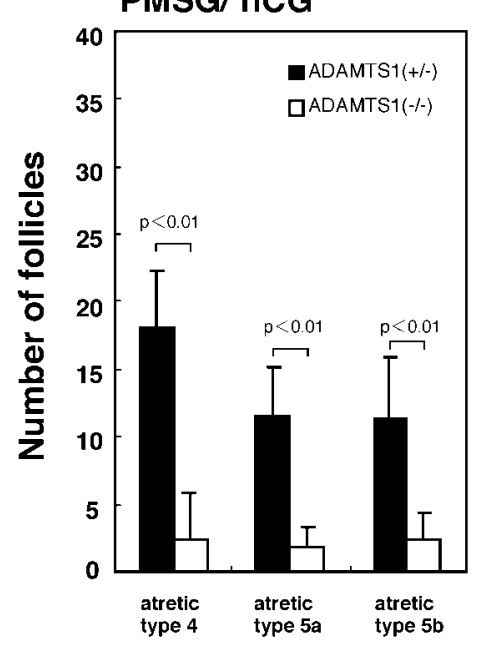

environment. The increases in early-stage follicles (type 4) may be attributable to impairment of follicular growth from the type 4 stage to advanced stages. PMSGinduced follicular growth may explain the relative decrease in the number of type 4 follicles observed in mice treated with PMSG alone (Fig. 2G).

\section{Reduction in general atretic pathways in ADAMTS-1 null mice}

To examine whether follicular atresia explains the decreased number of growing follicles, we analyzed changes in atretic follicles in ADAMTS- 1 null ovaries. As shown in Fig. 3B and $\mathrm{C}$, the number of atretic follicles from preantral follicles (types $4,5 \mathrm{a}$, and $5 \mathrm{~b}$ ) was markedly lower in ADAMTS-1 null mice treated with PMSG or PMSG/hCG than in control mice. Similarly, under the unstimulated condition, a significant reduction in the number of atretic follicles derived from preantral follicles was observed in ADAMTS-1 null ovaries (Fig. 3A). These data do not support the notion that enhanced atresia causes the reduction in healthy follicles in ADAMTS-1 null ovaries.

\section{Emergence of unusual follicles not associated with granulosa cell layers in ADAMTS-1 null ovaries}

Further detailed analysis of ovarian histology revealed that a number of unusual follicles without cytoplasmic fragmentation and not surrounded by layers of normal-looking granulosa cells appeared in unstimulated ADAMTS-1 null ovaries, while such unusual follicles were rarely seen in the controls (Fig. 4A and C). These were not classified as atretic follicles based on the criteria that we used in this study because there were no apparent fragmentation of oocytes, a sign of authentic follicular atresia. These unusual follicles were clearly delineated from usual atresia observed in control ovaries (Fig.4A and B). The number of these unusual follicles in ADAMTS-1 null mice was not dependent on exogenous gonadotropin stimulation (Fig. 4C). These unusual oocytes were larger than oocytes of healthy type 3 follicles, and approximately equivalent in size to oocytes of type 4 follicles (Fig. 4A). Therefore, such unusual

Figure 3 Reduction in atretic follicles from preantral stages in ADAMTS-1 null mice. (A-C) The numbers of atretic follicles from preantral stages in ovaries of ADAMTS-1 -/- and +/- mice treated with PMSG $(B)$ or PMSG/hCG $(C)$, and without gonadotropin (A). ADAMTS-1 //- and ADAMTS-1 +/- mice were treated with PMSG (48 h) ( $n=5$ for each group) (B), or PMSG (48 h) /hCG (15 h) ( $n=6$ for each group) (C), and without gonadotropin ( $n=5$ for each group) (A), followed by excision of ovaries, which were then histologically analyzed. The results are expressed as follicles per ovary. The data are means \pm S.D. 
$(A)$

ADAMTS1 (-/-)

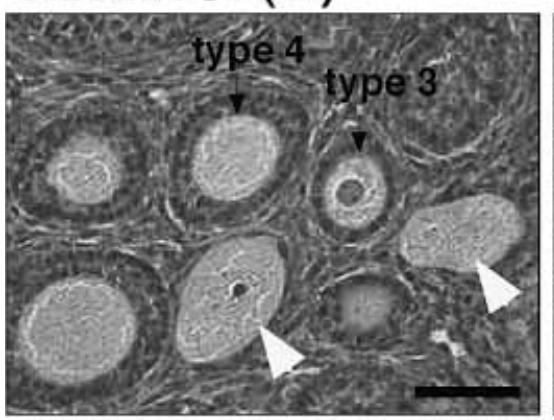

(B)

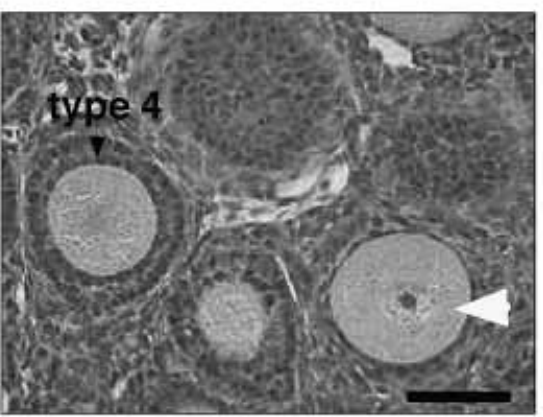

ADAMTS1 (+/-)

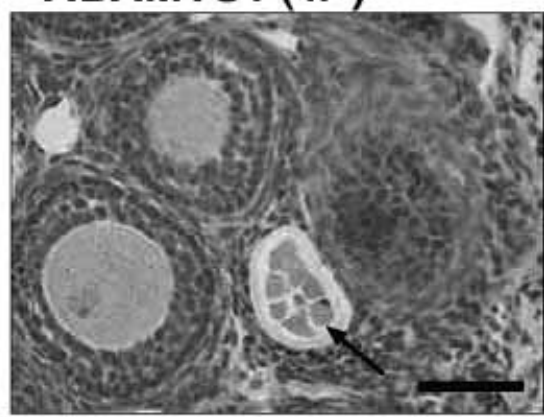

(C)

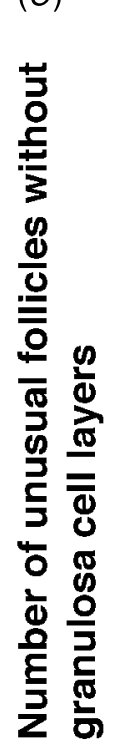

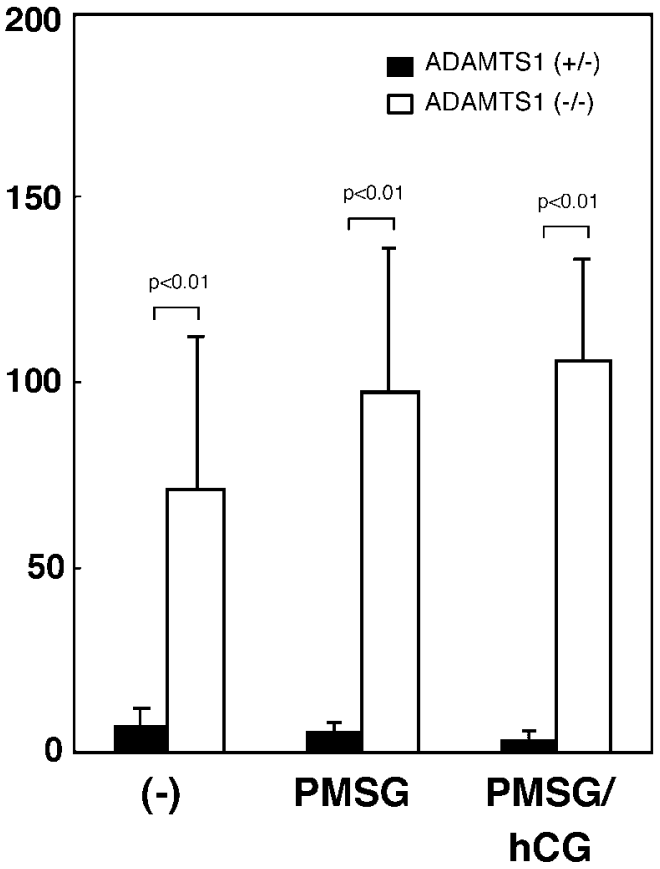

follicles might be formed from follicles at type 4 or later preantral stages by losing their granulosa cell layers in ADAMTS-1 null ovaries. ADAMTS-1 may be required for maintenance of follicle structure at the preantral stages.

\section{Increased rate of follicles failing to ovulate in ADAMTS-1 null mice}

The role of ADAMTS-1 in the ovulatory process was evaluated by determining the proportion of total anovulatory follicles (UF+LUF) as a percentage of the total mature follicles (UF+LUF+CL). As seen in Fig. 5A, the percentage of anovulatory follicles significantly increased in ADAMTS-1 null mice treated with PMSG /hCG when compared with control mice, indicating
Figure 4 Appearance of a number of unusual follicles without granulosa cell layers in ADAMTS-1 null ovaries. (A) Unusual follicles without granulosa cell layers (indicated by arrowheads) formed in ADAMTS-1 null ovaries without stimulation. Healthy follicles (types 3 and 4) are also indicated. Scale bar: $100 \mu \mathrm{m}$. (B) An atretic follicle (arrow) observed in control ovaries without stimulation. Scale bar: $100 \mu \mathrm{m}$. (C) Comparison of the number of unusual follicles in ADAMTS-1 -/- and ADAMTS-1 +/- mice treated with PMSG (48 hr)/hCG (15 h) ( $n=6$ for each group), or PMSG (48 h) ( $n=5$ for each group), and without gonadotropin ( $n=5$ for each group). The results are expressed as follicles per ovary. The data are means \pm S.D.

that ovulatory ability is significantly compromised in ADAMTS-1 null females. The increase in the anovulatory follicle rate in ADAMTS-1 null mice was attributable to an increase in UF but in LUF (Fig. 5B and G). In 20 of 22 UFs found in ADAMTS- 1 null ovaries, the cumulus cells had already expanded around the oocytes (Fig. 6B, D and E), thus suggesting that COC expansion is not affected. In this respect, UF in ADAMTS-1 null mice resemble preovulatory follicles, except for degeneration of the oocyte and the zona pellucida (Fig. 6B and D). In addition, partial luteinizing changes were sporadically observed in the granulosa layer in UF in ADAMTS-1 null ovaries (Fig. 6F) and UF in ADAMTS-1 null mice often retained the thick theca layer despite hGG treatment (Fig. 6E). These findings indicate that thinning and rupture of the follicle wall 

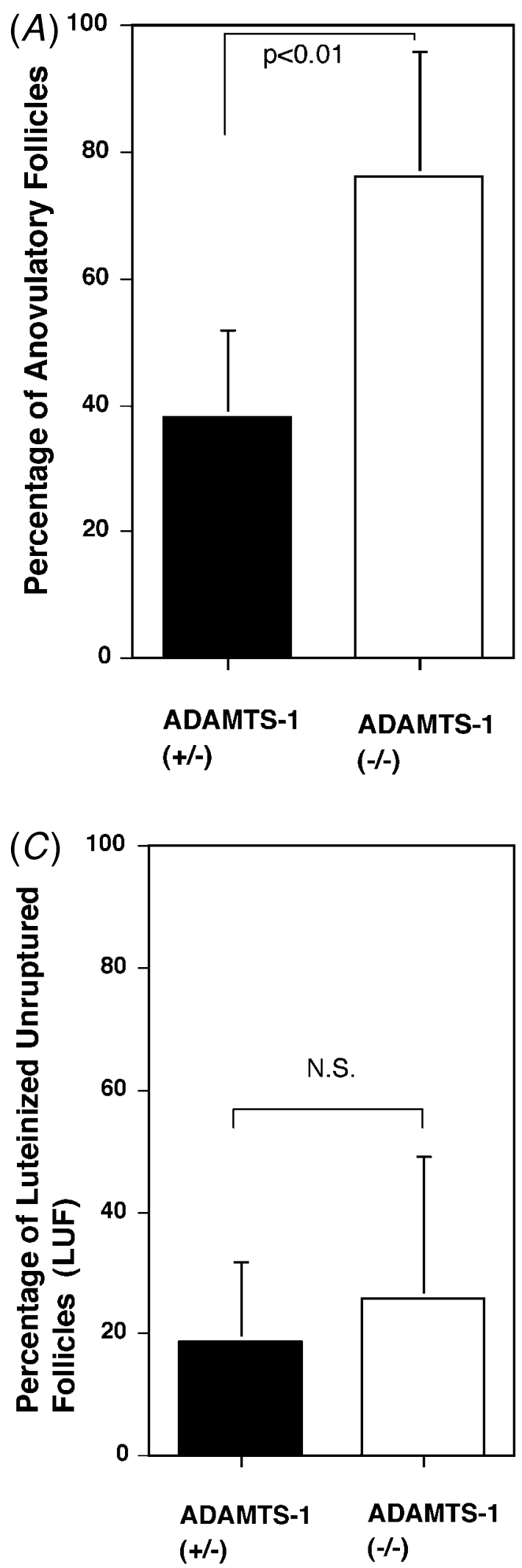

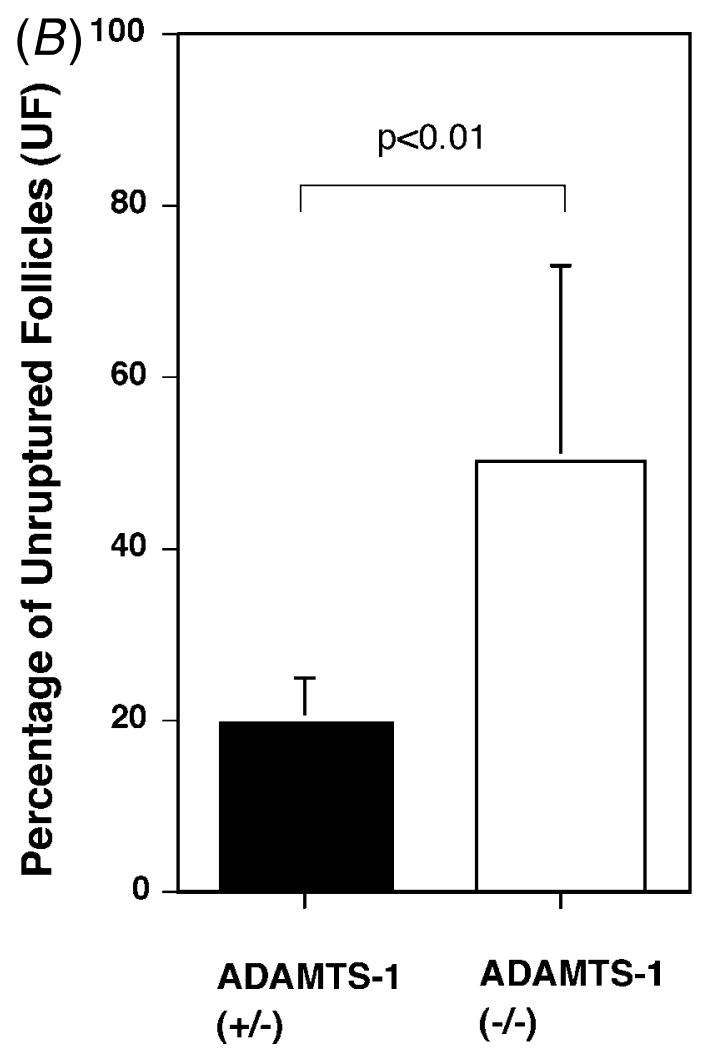

Figure 5 Involvement of ADAMTS-1 in the ovulatory process. (A-C) Comparison of percentages of anovulatory follicles (A), UF (B), and LUF (C) in ADAMTS-1 -/- and ADAMTS-1 +/- mice. ADAMTS-1 -/$(n=7)$ and ADAMTS-1 +/- $(n=7)$ mice were treated with PMSG followed by hCG $48 \mathrm{~h}$ later, $15 \mathrm{~h}$ after which the ovaries were excised and histologically analyzed as described in Materials and methods. Percentages of anovulatory follicles were calculated according to the following formula: anovulatory follicles

(UF+LUF)/total-mature follicles (UF+LUF+CL). The results are expressed as follicles per ovary. The data are means \pm S.D. 


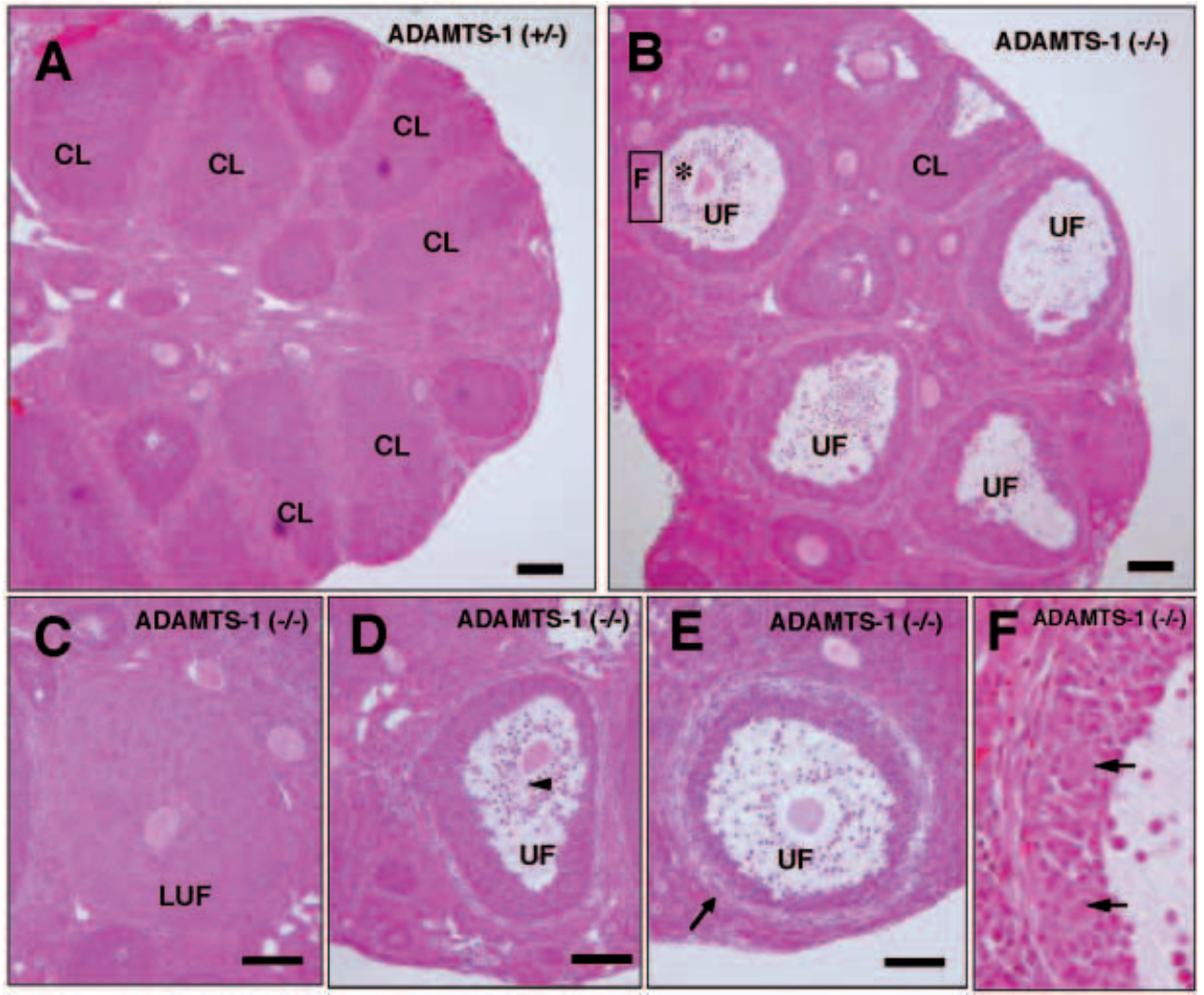

Figure 6 Ovarian histology of ADAMTS-1 homozygous mutant females. Sections of ovaries from ADAMTS-1 +/- mice (A), and ADAMTS-1 -/- mice (B-F) treated with PMSG/hCG (15 h) were stained with hematoxylin-eosin. Expansion of the cumulous cells was observed in UF of ADAMTS-1 -/females (B, D and E). (B) UF of ADAMTS-1 -/- mice having a degenerated oocyte (asterisk). (C) typical LUF. (D) UF containing a degenerated zona pellucida. (E) UF surrounded by a thick theca cell layer (arrow). Scale bar: $100 \mu \mathrm{m}$. (F) High-magnification portion of panel (B), depicting partial luteinizing changes (arrow) in the granulosa cell layer of UF in ADAMTS-1 null ovaries.

are compromised in ADAMTS-1 null mice and that luteinization, albeit incomplete, proceeded in the resultant follicles.

\section{Dysplasitic vasculature of ADAMTS-1 null ovaries}

Alteration of ovarian blood vessels in ADAMTS-1 null mice was examined by immunohistochemical staining with anti-CD31 antibody. In preantral (type 5b) and small antral follicles (type 6), a single-layer capillary network was observed in the theca layer in ADAMTS-1 null ovaries (Fig. 7B and D), as in control mice (Fig. 7A and $\mathrm{C}$ ). In contrast, the vasculature of large follicles in ADAMTS-1 null mice was somewhat different from that in control mice. Large follicles in ADAMTS-1 null ovaries often had additional layers of capillary network outside the capillary plexus in the theca layer (Fig. 7G and $\mathrm{H}$ ), while most large follicles in control mice had only a single capillary layer (Fig. 7E). In ADAMTS-1 null ovaries, 10 of 18 large follicles had two layers of vascular plexus, while in control ovaries, this number was 3 of $28, P<0 \cdot 01$.

Dysplastic vasculature was more striking in the ovarian medulla in ADAMTS-1 null mice (Fig. 8). Under super-ovulatory conditions, various sizes of blood vessels, including a number of large blood vessels (50-150 $\mu \mathrm{m}$ in diameter), were formed in the medullar zone of control ovaries (Fig. 8A and G). Large blood vessels (more than $50 \mu \mathrm{m}$ in diameter) were very few in ovaries of unstimulated control mice, and formation of large blood vessels was induced by administration of gonadotropins in control ovaries (Fig. 8F). In contrast, ADAMTS-1 null ovaries had a small medullar zone (Fig. 8B and E). Formation of large blood vessels of the medullary zone in the presence of gonadotropins (PMSG or PMSG/hCG) was significantly impaired in ADAMTS-1 null mice although smaller vessels were present (Fig. 8B, D, and F). These observations demonstrate that the vascular network of the ovarian medulla is dysplastic or disorganized in ADAMTS-1 null mice. 

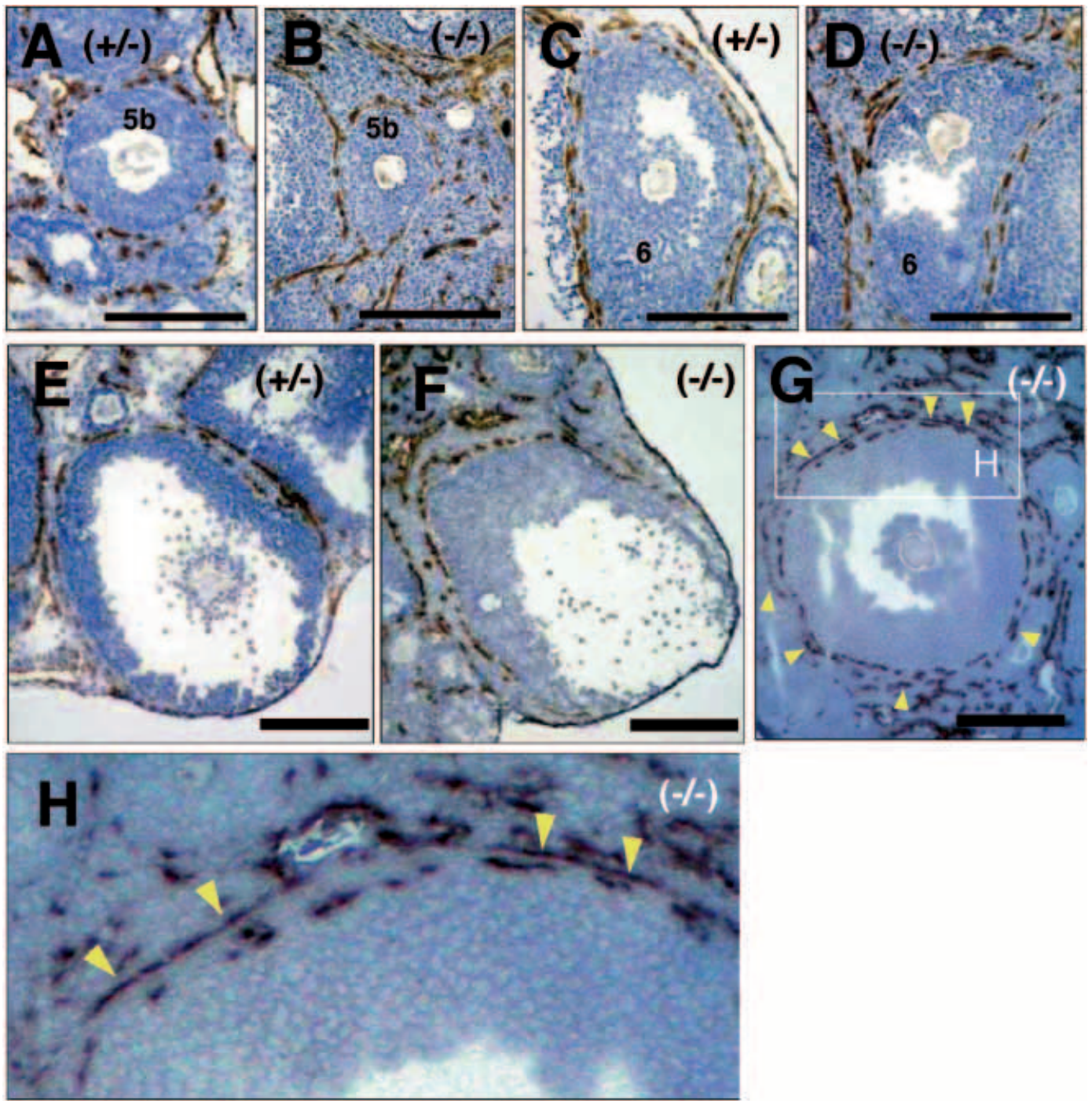

Figure 7 Follicular microvasculature of ADAMTS-1 null ovaries. Sections of ovaries from ADAMTS-1 +/- (A, C, and $\mathrm{E})$, and ADAMTS-1 -/- mice (B, D, F, G and H) treated with PMSG/hCG (11 h) were stained immunohistochemically with anti-mouse CD31 antibody. (A and B) type 5b follicles; $(C$ and $D)$ type 6 follicles; $(E$ and $F$ ) preovulatory follicles; $(G)$ follicles with a single antrum. $(H)$ High-magnification portion of panel $(G)$. In $\mathrm{G}$ and $\mathrm{H}$, yellow arrowheads indicate additional capillary layers outside the capillary plexus in the theca layer of follicles in ADAMTS-1 null ovaries. Counterstain: hematoxylin. Scale bar: $200 \mu \mathrm{m}$.

\section{Discussion}

Mouse knockout models have provided critical information on the factors associated with ovarian follicular development (Dong et al. 1996, Dierich et al. 1998, Couse et al. 1999, Soyal et al. 2000) and the ovulatory process (Lydon et al. 1995, Hizaki et al. 1999, Davis et al. 1999). We and others have shown that ADAMTS-1 plays important roles in the functions of female genital organs by analyzing ADAMTS-1 null mice (Shindo et al. 2000, Mittaz et al. 2004). In the present study, we demonstrate that abrogation of ADAMTS-1 impaired ovulation of oocytes at two different levels; in the development of growing follicles and in the ovulatory process of mature follicles in response to gonadotropins.

Ovarian folliculogenesis starts with the recruitment of nongrowing primordial follicles in the perinatal stage, and proceeds through the primary follicle, preantral, and antral stages (Matzuk et al. 2002), under the influence of various factors including FSH, estrogen and growth factors such as c-kit and GDF-9 (Dong et al. 1996, Dierich et al. 1998, Couse et al. 1999, Donovan \& de Miguel 2001, Matzuk et al. 2002). This study demonstrated that ADAMTS-1 is also required for complete 

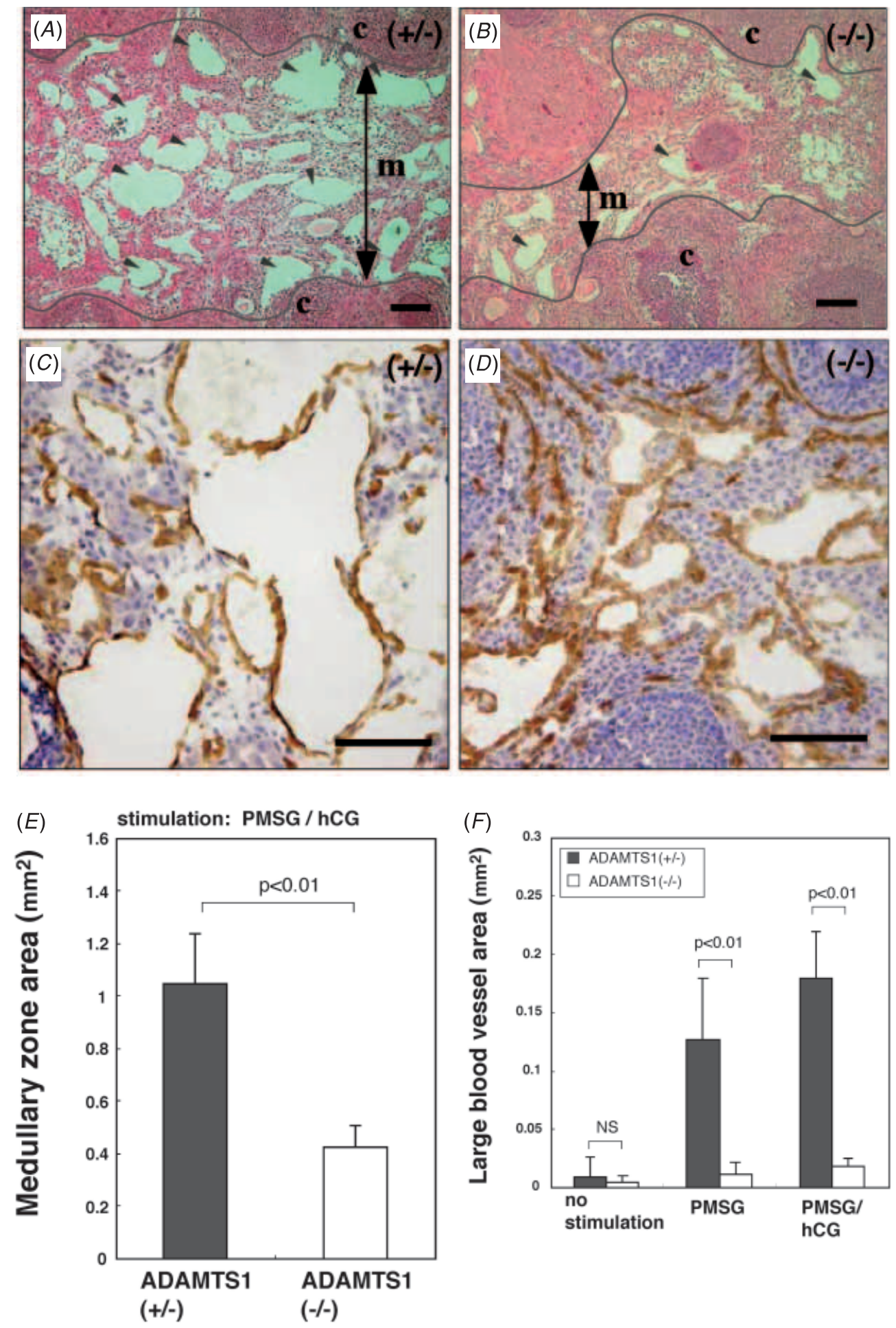
folliculogenesis. In ADAMTS-1 null mice, the number of type 4 follicles was increased when compared with controls, whereas the number of type $5 \mathrm{~b}$ follicles and later stages were decreased. Instead of healthy preantral follicles at type $5 \mathrm{~b}$ or later, unusual follicles containing enlarged oocytes without fragmentation and losing normal granulosa cells, probably originating from an unusual atretic process in type 4 or 5 follicles, were elevated in the ADAMTS-1 ovaries. Atresia was not accelerated in preantral follicles. These findings are compatible with the notion that ADAMTS-1 is important for follicles to autonomously develop beyond the type 4 or $5 \mathrm{a}$ into the next stage and that abrogation of ADAMTS-1 leads to formation of unusual atretic follicles without granulosa cell layers. Granulosa cells rapidly proliferate from the preantral follicle stage (type 4 and 5a), and the ECM in the granulosa cell layer must be reconstituted during follicular development. Thus, ADAMTS-1 may be involved in folliculogenesis as well as the maintenance of follicular structure by participating in the remodeling of the ECM surrounding the granulosa cells of growing follicles.

Ovulation initiated by the LH surge consists of several sequential events involving enlargement of the antrum, expansion of the COC, degradation of the follicle and ovarian wall at the apex of the mature follicles, and release of the COG (Tsafriri et al. 1996, Murdoch 2000, Richards et al. 2002). We found that the proportion of anovulatory follicles, specifically of UF to total mature follicles, was significantly higher in the ovaries of ADAMTS-1 null mice treated with PMSG/hCG. Significant induction of ADAMTS-1 mRNA expression in rats and in mice has been shown to occur in the granulosa layer of the large follicles after hCG administration (Espey et al. 2000, Robker et al. 2000). A more recent study demonstrated that ADAMTS-1 protein predominantly accumulates in the ECM of the COG during cumulus cell expansion after hCG administration (Russell et al. 2003). Russell et al. (2003) also reported that processing of versican is reduced in COC of Progesterone receptor knockout mice in which ADAMTS-1 expression is downregulated (Russell et al. 2003). It has therefore been hypothesized that ADAMTS-1 is involved in the re-organization of the
COC matrix by modulating versican prior to hCGinduced ovulation. We observed that cumulus cell expansion occurred in most of the unruptured large follicles of PMSG/hCG-treated ADAMTS-1 null ovaries, thus indicating that ADAMTS- 1 is not essential for cumulus cell expansion. However, other ADAMTS family members, such as ADAMTS-4 which has similar substrate specificity to ADAMTS-1, may compensate for the loss of the function of ADAMTS- 1 in cumulus expansion; it has been shown that ADAMTS family members including ADAMTS- 4 and -5 are expressed in preovulatory follicles in the ovary (Madan et al. 2003, Russell et al. 2003). Similarly, the incomplete blockage of the ovulatory process observed in ADAMTS-1 knockout mice might be due to the compensatory functions of other ADAMTS family members, such as ADAMTS-4 and -5 .

Degradation of ECM within the theca layer and tunica albuginea is necessary for follicle rupture. Matrix metalloproteinases (MMPs), such as MMP13, have been suggested to contribute to the breakdown of the follicle wall (Curry et al. 2001). We found that ADAMTS-1 null mice exhibited impairment of theca layer thinning and the ovulatory response to PMSG/hGG treatment (Fig. 6). Although ADAMTS-1 is not capable of degrading fibrillar collagens, such as collagens I and III (Rodriguez-Manzaneque et al. 2002), it is possible that ADAMTS-1 indirectly promotes degradation of the collagenous layers of the follicle wall by triggering signals that activate MMPs. Our previous study showed that ADAMTS-1 null mice exhibit renal phenotypes that resemble human UPJ stenosis, accumulating excessive collagen fibers in UPJ tissue (Shindo et al. 2000). It is likely that ADAMTS-1 plays a similar role in regulation of EGM remodeling in both UPJ tissue and ovaries.

Vascular endothelial growth factor (VEGF)-mediated follicular angiogenesis is involved in gonadotropindependent follicle development (Zimmermann et al. 2003). As ADAMTS-1 generally functions as an antiangiogenic factor through inhibition of VEGF action, it can be expected that loss of ADAMTS-1 may affect vasculature in the ovary. In the present study, we found that the follicular angiogenesis of large follicles was somehow upregulated in ADAMTS-1 null ovaries. This

Figure 8 Medullary vascular structure of ADAMTS-1 null ovaries. (A and B) Histology of the medullar zone. Sections of ovaries from ADAMTS-1 +/- (A) and ADAMTS-1 -/- mice (B) treated with PMSG/hCG (15 h) were stained with hematoxylin-eosin. Arrowheads indicate large blood vessels (more than $50 \mu \mathrm{m}$ in diameter). Boundaries between the medullar zone (m) and the cortical zone (c) are indicated by the gray lines. Scale bar: $100 \mu \mathrm{m}$. (C and D) Immunohistochemistry for CD31 in the medullar zone. Sections of ovaries from ADAMTS-1 +/- (C) and ADAMTS-1 -/- mice (D) treated with PMSG/hCG (11 h) were stained immunohistochemically with anti-mouse CD31 antibody. Counterstaining: hematoxylin. Scale bar: $100 \mu \mathrm{m}$. (E) Reduction in the medullary zone area in ADAMTS-1 null ovaries treated with PMSG/hCG $(15 \mathrm{~h})$. The areas of the central medullary zones of ADAMTS-1 +/- $(n=6)$ and ADAMTS-1 -/- $(n=6)$ ovaries, which are shown in panels $(A)$ and $(B)$, were measured as described in Materials and methods. (F) Reduction in the area occupied by large blood vessels (more than $50 \mu \mathrm{m}$ in diameter) in ADAMTS-1 null ovaries treated with PMSG (48 h) ( $n=5$ for each group), or PMSG/hCG (15 h) ( $n=6$ for each group), and without gonadotropin ( $n=5$ for each group). Total area occupied by large vessels in the central medullary zones was measured as described in Materials and methods. 
might be due to loss of anti-angiogenic activity of ADAMTS-1. In contrast, more significant changes in the vasculature network in ADAMTS-1 null ovaries were observed in the medullary zone. We found that gonadotropin-induced formation of large blood vessels in the medullary zone was significantly impaired in ADAMTS-1 null mice. This shows that ADAMTS-1 does not function merely as an anti-angiogenic factor in the ovary, but positively regulates the formation of the blood vessel network, although the mechanisms remain to be investigated. ADAMTS-1 might participate in branching and dilation processes of blood vessel formation, although we cannot exclude the possibility that vascular changes in the medullary zone are the result of a decreased number of mature follicles.

As we found that types $5 \mathrm{~b}$ and 6 follicles in ADAMTS-1 null mice had normal vascular plexus, it is unlikely that the decreased follicular development of ADAMTS-1 null mice can be attributed to alteration in follicular angiogenesis. However, dysplasia of large blood vessels in the medullary zone may affect blood supply to growing follicles and, therefore retard gonadotropindependent follicular development in ADAMTS-1 null mice.

In summary, our results provide evidence that ADAMTS-1 is involved in the ovulatory response of mature follicles, follicular development, and the organization of the medullary vascular network. An interesting question regarding the human ovary that arises based on the present results is whether defective ADAMTS-1 expression is a model for any ovarian dysfunction observed in human patients. Further studies on the functions of ADAMTS family members, including ADAMTS-1, in the ovary can be expected to advance the understanding of the molecular mechanisms of infertility.

\section{Acknowledgements}

After completion of this work concerning ovulation and folliculogenesis, Mittaz et al. reported that ovulation was impaired in ADAMTS-1 null mice (2004). We are grateful to Dr K Murakami (Kanazawa University) for helpful discussion and to Mses A Katayama, M Kaida and M Watanabe (Kanazawa University) for their technical assistance.

\section{Funding}

This work was supported by a Grant-in-Aid from the Ministry of Education, Science, and Culture of Japan. The authors declare that there is no conflict of interest that would prejudice the impartiality of this work.

\section{References}

Boerboom D, Russell DL, Richards JS \& Sirois J 2003 Regulation of transcripts encoding ADAMTS-1 (a disintegrin and metalloproteinase with thrombospondin-like motifs-1) and progesterone receptor by human chorionic gonadotropin in equine preovulatory follicles. Fournal of Molecular Endocrinology 31 $473-485$.

Couse JF, Hewitt SC, Bunch DO, Sar M, Walker VR, Davis BJ \& Korach KS 1999 Postnatal sex reversal of the ovaries in mice lacking estrogen receptors $\alpha$ and $\beta$. Science 286 2328-2331.

Curry TE, Jr. \& Osteen KG 2001 Cyclic changes in the matrix metalloproteinase system in the ovary and uterus. Biology of Reproduction 64 1285-1296.

Davis BJ, Lennard DE, Lee CA, Tiano HF, Morham SG, Wetsel WC \& Langenbach R 1999 Anovulation in cyclooxygenase2-deficient mice is restored by prostaglandin $\mathrm{E}_{2}$ and interleukin-1 ß. Endocrinology $1402685-2695$.

Dierich A, Sairam MR, Monaco L, Fimia GM, Gansmuller A, LeMeur M \& Sassone-Corsi P 1998 Impairing follicle-stimulating hormone (FSH) signaling in vivo: Targeted disruption of the FSH receptor leads to aberrant gametogenesis and hormonal imbalance. PNAS 95 13612-13617.

Dong J, Albertini DF, Nishimori N, Kumar TR, Lu N \& Matzuk M 1996 Growth differentiation factor-9 is required during early ovarian folliculogenesis. Nature 383 531-535.

Donovan PJ \& de Miguel MP 2001 The role of the c-kit/kit ligand axis in mammalian gametogenesis. In Transgenics in Endocrinology, pp 147-163. Eds M Matzuk, CW Brown \& TR Kumar. New Jersey: Humana Press.

Espey LL, Yoshioka S, Russell DL, Robker RL, Fujii S \& Richards JS 2000 Ovarian expression of a disintegrin and metalloproteinase with thrombospondin motifs during ovulation in the gonadotropinprimed immature rat. Biology of Reproduction 62 1090-1095.

Hizaki H, Segi E, Sugimoto T, Hirose M, Saji T, Ushikubi F, Matsuoka T, Noda Y, Tanaka T, Yoshida N, et al. 1999 Abortive expansion of the cumulus and impaired fertility in mice lacking the prostaglandin E receptor subtype $\mathrm{EP}_{2}$. PNAS 96 10501-10506.

Iruela-Arispe ML, Carpizo D \& Luque A 2003 ADAMTS1: a matrix metalloprotease with angioinhibitory properties. Annals of the New York Academy of Sciences 995 183-190.

Kuno K \& Matsushima K 1998 ADAMTS-1 protein anchors at the extracellular matrix through the thrombospondin type I motifs and its spacing region. Fournal of Biological Chemistry 273 13912-13917.

Kuno K, Kanada N, Nakashima E, Fujiki F, Ichimura F \& Matsushima K 1997a Molecular cloning of a gene encoding a new type of metalloproteinase-disintegrin family protein with thrombospondin motifs as an inflammation associated gene. Journal of Biological Chemistry 272 556-562.

Kuno K, Iizasa H, Ohno S \& Matsushima K 1997b The exon/intron organization and chromosomal mapping of the mouse ADAMTS-1 gene encoding an ADAM family protein with TSP motifs. Genomics 46 466-471.

Kuno K, Terashima Y \& Matsushima K 1999 ADAMTS-1 is an active metalloproteinase associated with the extracellular matrix. Fournal of Biological Chemistry 274 18821-18826.

Kuno K, Okada Y, Kawashima H, Nakamura H, Miyasaka M, Ohno H \& Matsushima K 2000 ADAMTS-1 cleaves a cartilage proteoglycan, aggrecan. FEBS Letters 478 241-245.

Kuno K, Bannai K, Hakozaki M, Matsushima K \& Hirose K 2004 The carboxyl-terminal half region of ADAMTS-1 suppresses both tumorigenicity and experimental tumor metastatic potential. Biochemical Biophysical Research Communications 319 1327-1333.

Luque A, Carpizo DR \& Iruela-Arispe ML 2003 ADAMTS1/METH1 inhibits endothelial cell proliferation by direct binding and sequestration of VEGF165. Fournal of Biological Chemistry 278 23656-23665. 
Lydon JP, DeMayo FJ, Funk CR, Mani SK, Hughes AR, Montgomery CA, Shyamala G, Conneely OM \& O'Malley BW 1995 Mice lacking progesterone receptor exhibit pleiotropic reproductive abnormalities. Genes and Development 9 2266-2278.

Madan P, Bridges PJ, Komar CM, Beristain AG, Rajamahendran R, Fortune JE \& MacCalman CD 2003 Expression of messenger RNA for ADAMTS subtypes changes in the periovulatory follicle after the gonadotropin surge and during luteal development and regression in cattle. Biology of Reproduction 69 1506-1514.

Matzuk MM, Burns KH, Viveiros MM \& Eppig JJ 2002 Intracellular communication in the mammalian ovary: oocytes carry the conversation. Science 296 2178-2180.

Mittaz L, Russell DL, Wilson T, Brasted M, Tkalcevic J, Salamonsen LA, Hertzog PJ \& Pritchard MA 2004 Adamts-1 is essential for the development and function of the urogenital system. Biology of Reproduction 70 1096-1105.

Morita Y, Perez GI, Maravei DV, Tilly KI \& Tilly JL 1999 Targeted expression of bcl-2 in mouse inhibit ovarian follicle atresia and prevent spontaneous and chemotherapy induced oocyte apoptosis in vitro. Molecular Endocrinology 13 841-850.

Murdoch WJ 2000 Proteolytic and cellular death mechanisms in ovulatory ovarian rupture. Biological Signals and Receptors 9 102-114.

Pedersen T \& Peters H 1968 Proposal for a classification of oocytes and follicles in the mouse ovary. Fournal of Reproduction and Fertility 17 555-557.

Richards JS, Russell DL, Ochsner S \& Espey LL 2002 Ovulation: new dimensions and new regulators of the inflammatory-like response. Annual Review of Physiology 64 69-92.

Robker RL, Russell DL, Espey LL, Lydon JP, O’Malley BW \& Richards JS 2000 Progesterone-regulated genes in the ovulation process: ADAMTS-1 and cathepsin L proteases. PNAS 97 4689-4694.

Rodriguez-Manzaneque JC, Westling J, Thai SN, Luque A, Knauper V, Murphy G, Sandy JD \& Iruela-Arispe ML 2002 ADAMTS1 cleaves aggrecan at multiple sites and is differentially inhibited by metalloproteinase inhibitors. Biochemical Biophysical Research Communications 293 501-508.

Russell DL, Doyle KM, Ochsner SA, Sandy JD \& Richards JS 2003 Processing and localization of ADAMTS-1 and proteolytic cleavage of versican during cumulus matrix expansion and ovulation. Fournal of Biological Chemistry 278 42330-42339.

Sandy JD, Westling J, Kenagy RD, Iruela-Arispe ML, Verscharen C, Rodriguez-Mazaneque JC, Zimmermann DR, Lemire JM, Fischer JW, Wight TN \& Clowes AW 2001 Versican V1 proteolysis in human aorta in vivo occurs at the Glu441-Ala442 bond, a site that is cleaved by recombinant ADAMTS-1 and ADAMTS-4. Fournal of Biological Chemistry 276 13372-13378.

Shindo T, Kurihara H, Kuno K, Yokoyama H, Wada T, Kurihara Y, Imai T, Wang Y, Ogata M, Nishimatsu H, et al. 2000 ADAMTS-1: a metalloproteinase-disintegrin essential for normal growth, fertility, and organ morphology and function. Fournal of Clinical Investigation 105 1345-1352.

Soyal SM, Amleh A \& Dean J 2000 FIG $\alpha$, a germ cell-specific transcription factor required for ovarian follicle formation. Development 127 4645-4654.

Tsafriri A \& Chun S-Y 1996 Ovulation. In Reproductive Endocrinology, Surgery, and Technology. pp 235-249. Eds EY Adashi \& Z Rosenwaks. Philadelphia: Lippincott-Raven.

Vazquez F, Hastings G, Ortega MA, Lane TF, Oikemus S, Lombardo M \& Iruela-Arispe ML 1999 METH-1, a human ortholog of ADAMTS-1, and METH-2 are members of a new family of proteins with angio-inhibitory activity. Fournal of Biological Chemistry 274 23349-23357.

Zimmermann RC, Hartman T, Kavic S, Pauli SA, Bohlen P, Sauer MV \& Kitajewski J 2003 Vascular endothelial growth factor receptor 2-mediated angiogenesis is essential for gonadotropin-dependent follicle development. Fournal of Clinical Investigation 112 659-669.

Received in final form 16 June 2005

Accepted 22 June 2005

Made available online as an Accepted Preprint 14 July 2005 